\title{
Fixed point theorems on ordered vector spaces
}

\author{
Jin Lu Li ${ }^{1 \dagger}$, Cong Jun Zhang ${ }^{2 \dagger}$ and Qi Qiong Chen ${ }^{3 \dagger}$
}

${ }^{\text {*Correspondence: }}$ qiqiongchen@163.com

${ }^{3}$ Department of Applied Mathematics, Nanjing University of Science and Technology, Xiaolingwei Street, Nanjing, Jiangsu 210094, China

†Equal contributors

Full list of author information is available at the end of the article

\begin{abstract}
In this paper, we introduce the concept of ordered metric spaces with respect to some ordered vector spaces, which is an extension of the normal metric spaces. Then we investigate some properties of ordered metric spaces and provide several fixed point theorems. As applications we prove several existence theorems for best ordered approximation.
\end{abstract}

MSC: 46B42; 47H10; 58J20; 91A06; 91A10

Keywords: ordered vector spaces; order-continuity; ordered Lipschitz condition; order-preserving mapping; best ordered approximation; fixed point

\section{Introduction}

Let $S$ be a nonempty set and let $X$ be a Banach space. Let $K$ be a closed convex cone in $X$. The cone metric defined on $S$, with respect to the cone $K$ in $X$, is a bifunction $d: S \times S \rightarrow X$, for which the following properties hold:

(a) $d(u, v) \in K$ and $d(u, v)=0$ if and only if $u=v$;

(b) $d(u, v)=d(v, u)$;

(c) $d(u, s)+d(s, v)-d(u, v) \in K$, for all $s, u, v \in S$.

Note that if we take $X$ to be the set of real numbers and take $K=[0, \infty)$, then any cone metric space with respect to $K$ turns out to be a normal metric space. Hence the concept of vector metric spaces extends the notion of the normal metric spaces.

In the field of nonlinear analysis, vector metric spaces have been widely studied by many authors (see [1]). Similarly to ordinary analysis, the concepts of continuity and of the Lipschitz condition for mapping on vector metric spaces have been introduced and have been applied for proving the existence of fixed points (see [2-4]).

Since the definition of vector metrics is based on closed convex cones in normed vector spaces, and every closed convex cone in a vector space can induce a partial order on it, which equips this vector space to be an ordered vector space, it is natural to generalize the concept of cone metric spaces to ordered metric spaces (see Section 3).

This paper is organized as follows: in Section 2, we recall some concepts of ordered vector spaces and order-convergence of sequences; in Section 3, we introduce the concept of ordered metric spaces and investigate some properties; in Section 4, several fixed point theorems on ordered metric spaces are provided; in Section 5, we extend the concept of best ordered approximation and prove several existence theorems by applying the fixed point theorems provided in Section 4.

@2014 Li et al.; licensee Springer. This is an Open Access article distributed under the terms of the Creative Commons Attribution License (http://creativecommons.org/licenses/by/2.0), which permits unrestricted use, distribution, and reproduction in any medium, provided the original work is properly cited. 


\section{Preliminaries}

In this section, we recall some concepts of ordered sets and some properties of orderlimits. For more details, the readers are referred to [5-7]. Then we extend the concept of order-continuity of mappings on ordered vector spaces and provide some properties that are similar to those of ordinary limits in analysis. These properties will be frequently used throughout this paper.

In this paper, all vector spaces considered are real vector spaces. A vector space $X$ equipped with a partial order $\succcurlyeq^{X}$ (that is, $\succcurlyeq^{X}$ is a reflexive, antisymmetric, and transitive binary relation on $X$ ) is called a partially ordered vector space, or is simply called an ordered vector space, which is written as $\left(X, \succcurlyeq^{X}\right)$, if the following (order-linearity) properties hold:

(v1) $x \succcurlyeq^{X} y$ implies $x+z \succcurlyeq^{X} y+z$, for all $x, y, z \in X$.

(v2) $x \succcurlyeq^{X} y$ implies $\alpha x \succcurlyeq^{X} \alpha y$, for all $x, y \in X$ and $\alpha \geq 0$.

A sequence $\left\{x_{n}\right\}$ in an ordered vector space $\left(X, \succcurlyeq^{X}\right)$ is said to be order-decreasing, which is denoted by $x_{n} \downarrow$, whenever $m>n$ implies $x_{m} \preccurlyeq^{X} x_{n}$. An order-decreasing sequence $\left\{x_{n}\right\}$ is said to order-converge to $x$, if $x_{n} \downarrow$ and $\bigwedge\left\{x_{n}\right\}$ exists with $\bigwedge\left\{x_{n}\right\}=x$, which is denoted by $\left\{x_{n}\right\} \downarrow x$. The meaning of $x_{n} \uparrow$ is analogously defined for an order-increasing sequence $\left\{x_{n}\right\}$; and $\left\{x_{n}\right\} \uparrow x$, if and only if $x_{n} \uparrow$ and $\bigvee\left\{x_{n}\right\}$ exists with $\bigvee\left\{x_{n}\right\}=x$.

Lemma 2.1 Let $\left\{x_{n}\right\},\left\{y_{n}\right\}$ be two sequences in an arbitrary ordered vector space $\left(X, \succcurlyeq^{X}\right)$. The following properties hold:

1. $x_{n} \downarrow x$ implies $a x_{n} \downarrow$ ax, for every $a \geq 0$.

2. $x_{n} \downarrow x$ and $y_{n} \downarrow y$ imply $\left(x_{n}+y_{n}\right) \downarrow(x+y)$.

3. $x_{n} \downarrow x$ and $y_{n} \downarrow y$ imply $\left(a x_{n}+b y_{n}\right) \downarrow(a x+b y)$, for any nonnegative numbers $a$ and $b$.

Definition 2.2 An ordered vector space $\left(X, \succcurlyeq^{X}\right)$ is said to be generalized Archimedean if and only if for any given element $x \succcurlyeq^{X} 0$ and any decreasing sequence of positive numbers $\left\{a_{n}\right\}$ with limit 0 , we have

$$
a_{n} x \downarrow 0 .
$$

Let $X$ be a vector space and $K$ a closed convex cone in $X$. Define a binary relation $\succcurlyeq_{K}$ on $X$ as follows:

$$
x \succcurlyeq_{K} y \quad \text { if and only if } \quad x-y \in K, \quad \text { for every } x, y \text { in } X .
$$

Then $\succcurlyeq_{K}$ is a partial order on $X$, which satisfies conditions (v1) and (v2), and therefore $\left(X, \succcurlyeq_{K}\right)$ is an ordered vector space, which is said to be induced by the closed convex cone $K$.

Lemma 2.3 Every ordered vector space induced by a closed convex cone is generalized Archimedean.

The proof of Lemma 2.3 is straightforward and it is omitted here.

In order theory, the order completeness of a poset is as important as the topological counterpart of a topological space in analysis. Recall that a subset $C$ of an ordered vector space $\left(X, \succcurlyeq^{X}\right)$ is said to be chain complete if and only if for any chain $\left\{x_{\alpha}\right\}$ in $X, \bigvee\left\{x_{\alpha}\right\}$ exists. Next, we define a special case of chain-complete subsets. 
Definition 2.4 Let $\left(X, \succcurlyeq^{X}\right)$ be an ordered vector space and $C$ a subset of $X$. $C$ is said to be (sequentially) conditionally chain complete if and only if for any sequence $\left\{x_{n}\right\}$ in $C$, the following properties hold:

1. If $x_{n} \downarrow$ and $\left\{x_{n}\right\}$ has a lower bound, then $\bigwedge\left\{x_{n}\right\}$ exists satisfying $x_{n} \downarrow \bigwedge\left\{x_{n}\right\} \in C$.

2. If $x_{n} \uparrow$ and $\left\{x_{n}\right\}$ has an upper bound, then $\bigvee\left\{x_{n}\right\}$ exists satisfying $x_{n} \uparrow \bigvee\left\{x_{n}\right\} \in C$.

\section{Ordered metric spaces}

We extend the concept of cone metric to the following ordered metric with respect to ordered vector spaces.

Definition 3.1 Let $S$ be a nonempty set and let $\left(X, \succcurlyeq^{X}\right)$ be an ordered vector space. A bifunction $d_{X}: S \times S \rightarrow X$ is called an ordered metric on $S$, with respect to $X$ if, for every $u$, $v$, and $s$ in $S$, it satisfies the following conditions:

(m1) $d_{X}(u, v) \succcurlyeq^{X} 0$ with $d_{X}(u, v)=0$, if and only if $u=v$;

(m2) $d_{X}(u, v)=d_{X}(v, u)$;

(m3) $d_{X}(u, v) \preccurlyeq^{X} d_{X}(u, s)+d_{X}(s, v)$.

Then $\left(S, d_{X}\right)$ is called an ordered metric space, and $d_{X}(u, v)$ is called the ordered distance between $u$ and $v$, with respect to the ordered vector space $\left(X, \succcurlyeq^{X}\right)$.

We offer some examples below to demonstrate that the class of ordered metric spaces is a very broad one. First, note that $(R, \geq)$ is an ordered vector space with the ordinal real order $\geq$. Then we have the following results.

Example 3.2 The metric defined on a metric space is an ordered metric; and therefore, every metric space is an ordered metric space.

Example 3.3 Let $B$ be a Banach space with the norm $\|\cdot\|$ and let $d$ be the metric on $B$ induced by $\|\cdot\|$. Then $d$ is an ordered metric; and therefore, every Banach space with the metric induced by its norm is an ordered metric space (where the metric $d$ is defined by $d(u, v)=\|u-v\|$, for every $u, v \in B)$.

Example 3.4 Every cone metric on a nonempty set is an ordered metric; and therefore, every cone metric space is an ordered metric space.

As a matter of fact, if an ordered vector space $\left(X, \succcurlyeq_{K}\right)$ is induced by a closed convex cone $K$ in $X$, then from (1), $d(u, v) \in K$ if and only if $d_{X}(u, v) \succcurlyeq_{K} 0$; and therefore, the ordered metrics on sets extend the concept of cone metrics.

Example 3.5 Let $\left(X, \succcurlyeq^{X}\right)$ be a Riesz space (vector lattice). Then the bifunction $d_{X}: X \times$ $X \rightarrow X$ induced by the (ordered) absolute values on $X$ as $d_{X}(x, y)=|x-y|$, for every $x, y \in X$, is an ordered metric on $X$. Hence every Riesz space with the ordered metric induced by its (ordered) absolute values is an ordered metric space.

Here, as usual, for any $x \in X, x^{+}=x \vee 0, x^{-}=(-x) \vee 0$ and $|x|=x^{+} \vee x^{-}$are called the (ordered) positive part, negative part, and (ordered) absolute value of $x$, respectively.

In real analysis, an arbitrary metric on a metric space is a function with positive values in $R$, which is totally ordered with respect to the ordinal order of real numbers. This property implies that the distances of pairs of elements are comparable. But in ordered metric 
space, the ordered distances of pairs of elements are not always comparable. Thus, this idea provides us a useful tool to study some problems, which have outcomes in a poset.

Definition 3.6 A sequence $\left\{s_{n}\right\}$ in an ordered metric space $\left(S, d_{X}\right)$ is said to orderconverge to an element $s \in S$, which is denoted by $s_{n} o s$, whenever there exists another sequence $\left\{x_{n}\right\}$ in $\left(X, \succcurlyeq^{X}\right)$ with $x_{n} \downarrow 0$ such that

$$
d_{X}\left(s_{n}, s\right) \preccurlyeq^{X} x_{n} \text { holds, for each } n \text {. }
$$

In this case, $s$ is called an order-limit of the sequence $\left\{s_{n}\right\}$.

Lemma 3.7 If a sequence $\left\{s_{n}\right\}$ in an ordered metric space $\left(S, d_{X}\right)$ is order-convergent, then its order-limit is unique.

Proof Let $u$ and $v$ be order-limits of $\left\{s_{n}\right\}$. Then there are sequences $\left\{x_{n}\right\}$ and $\left\{z_{n}\right\}$ in $X$ with $x_{n} \downarrow 0$ and $z_{n} \downarrow 0$ such that

$$
d_{X}\left(s_{n}, u\right) \preccurlyeq^{X} x_{n} \quad \text { and } \quad d_{X}\left(s_{n}, v\right) \preccurlyeq^{X} z_{n}, \quad \text { for each } n .
$$

From condition (m3), they imply

$$
d_{X}(u, v) \preccurlyeq^{X} d_{X}\left(s_{n}, u\right)+d_{X}\left(s_{n}, v\right) \preccurlyeq^{X} x_{n}+z_{n}, \quad \text { for each } n \text {. }
$$

From Part 2 of Lemma 2.1, $\bigwedge\left(x_{n}+z_{n}\right)=0$. It implies $0 \preccurlyeq^{X} d_{X}(u, v) \preccurlyeq{ }^{X} 0$. Hence $d_{X}(u, v)=0$. From condition (m1) in the definition of ordered metric, it follows that $v=u$.

Definition 3.8 A sequence $\left\{s_{n}\right\}$ in an ordered metric space $\left(S, d_{X}\right)$ is called an orderCauchy sequence, whenever there exists another sequence $\left\{x_{n}\right\}$ in $\left(X, \succcurlyeq^{X}\right)$ with $x_{n} \downarrow 0$ such that

$$
d_{X}\left(s_{m}, s_{n}\right) \preccurlyeq{ }^{X} x_{n} \text { holds, for each } n \text {, and for every } m \geq n \text {. }
$$

Definition 3.9 An ordered metric space $\left(S, d_{X}\right)$ is said to be order-metric complete, whenever every order-Cauchy sequence in $S$ is order-convergent.

Definition 3.10 Let $\left(S, d_{X}\right)$ and $\left(T, d_{Y}\right)$ be ordered metric spaces, with respect to the ordered vector spaces $\left(X, \succcurlyeq^{X}\right)$ and $\left(Y, \succcurlyeq^{Y}\right)$, respectively. A mapping $f: S \rightarrow T$ is said to be sequentially or $\sigma$-continuous, whenever, for any sequence $\left\{s_{n}\right\} \subset S, s_{n} g s$, with respect to the ordered metric $d_{X}$ on $S$, implies $f\left(s_{n}\right) \stackrel{\rho}{\rightarrow} f(s)$, with respect to the ordered metric $d_{Y}$ on $T$.

\section{Several fixed point theorems on ordered metric spaces}

Theorem 4.1 Let $\left(S, d_{X}\right)$ be an order-metric complete ordered metric space, with respect to a generalized Archimedean ordered vector space $\left(X, \succcurlyeq^{X}\right)$. Let $f: S \rightarrow S$ be a self-mapping. Suppose there is a positive number $\alpha<1$, such that

$$
d_{X}\left(f\left(s_{1}\right), f\left(s_{2}\right)\right) \preccurlyeq^{X} \alpha d_{X}\left(s_{1}, s_{2}\right), \quad \text { for all } s_{1}, s_{2} \in S .
$$

Then $f$ has a unique fixed point. 
Proof Taking any $s_{0} \in S$, we define a sequence $\left\{f^{n}\left(s_{0}\right)\right\} \subset S$. From condition (4), it follows that

$$
d_{X}\left(f^{n+1}\left(s_{0}\right), f^{n}\left(s_{0}\right)\right) \preccurlyeq^{X} \alpha d_{X}\left(f^{n}\left(s_{0}\right), f^{n-1}\left(s_{0}\right)\right), \quad \text { for all positive integer } n \text {. }
$$

Iterating the above order-inequality yields

$$
d_{X}\left(f^{n+1}\left(s_{0}\right), f^{n}\left(s_{0}\right)\right) \preccurlyeq^{X} \alpha^{n} d_{X}\left(f\left(s_{0}\right), s_{0}\right), \quad \text { for all positive integer } n \text {. }
$$

By property $(\mathrm{m} 3)$ of order-metric and properties of partial orders in ordered vector spaces, for any positive integer $i$, we get

$$
\begin{aligned}
& d_{X}\left(f^{n+i}\left(s_{0}\right), f^{n}\left(s_{0}\right)\right) \preccurlyeq \alpha^{n+i} d_{X}\left(f\left(s_{0}\right), s_{0}\right)+\alpha^{n+i-1} d_{X}\left(f\left(s_{0}\right), s_{0}\right) \\
&+\cdots+\alpha^{n} d_{X}\left(f\left(s_{0}\right), s_{0}\right) \\
&= \alpha^{n}\left(\alpha^{i}+\alpha^{i-1}+\cdots+1\right) d_{X}\left(f\left(s_{0}\right), s_{0}\right) \\
& \preccurlyeq^{X} \frac{\alpha^{n}}{1-\alpha} d_{X}\left(f\left(s_{0}\right), s_{0}\right), \quad \text { for all positive integer } n .
\end{aligned}
$$

Take

$$
x_{n}=\frac{\alpha^{n}}{1-\alpha} d_{X}\left(f\left(s_{0}\right), s_{0}\right), \quad \text { for all positive integer } n .
$$

Since $\left(X, \succcurlyeq^{X}\right)$ is generalized Archimedean, from Definition 2.2, it yields $x_{n} \downarrow 0$. It implies that $\left\{f^{n}\left(s_{0}\right)\right\}$ is an order-Cauchy sequence in $\left(S, d_{X}\right)$, which is an order-metric complete ordered metric space. Hence, $\left\{f^{n}\left(s_{0}\right)\right\}$ has an order-limit, say $s^{*} \in C$; that is,

$$
f^{n}\left(s_{0}\right) \stackrel{o}{\rightarrow} s^{*}
$$

Then, from Definition 3.6, there is a sequence $\left\{y_{n}\right\} \subset X$, with $y_{n} \downarrow 0$ such that

$$
d_{X}\left(f^{n}\left(s_{0}\right), s^{*}\right) \preccurlyeq^{X} y_{n}, \quad \text { for each } n \text {. }
$$

Since the mapping $f: S \rightarrow S$ has the ordered Lipschitzian property given by (4) with $x_{n}$ defined in (5), from the above order inequality, it yields

$$
\begin{aligned}
d_{X}\left(f\left(s^{*}\right), s^{*}\right) & \preccurlyeq^{X} d_{X}\left(f^{n+1}\left(s_{0}\right), f\left(s^{*}\right)\right)+d_{X}\left(f^{n+1}\left(s_{0}\right), s^{*}\right) \\
& \preccurlyeq^{X} \alpha d_{X}\left(f^{n}\left(s_{0}\right), s^{*}\right)+d_{X}\left(f^{n+1}\left(s_{0}\right), s^{*}\right) \\
& \preccurlyeq^{X}(1+\alpha) y_{n}, \quad \text { for each } n .
\end{aligned}
$$

From Lemma 2.1, $y_{n} \downarrow 0$ implies $(1+\alpha) y_{n} \downarrow 0$. So we obtain

$$
0 \preccurlyeq^{X} d_{X}\left(f\left(s^{*}\right), s^{*}\right) \preccurlyeq^{X} \bigwedge\left\{(1+\alpha) y_{n}\right\}=0 .
$$

It implies

$$
d_{X}\left(f\left(s^{*}\right), s^{*}\right)=0 .
$$


It follows that $f\left(s^{*}\right)=s^{*}$; and therefore, $s^{*}$ is a fixed point of $f$. The uniqueness of the fixed point of $f$ immediately follows from condition (4) of the mapping $f$ and condition (m1) of the ordered metric.

From Example 3.5, any arbitrary Riesz space (vector lattice) $\left(X, \succcurlyeq^{X}\right)$ is an ordered metric space with the ordered metric induced by its (ordered) absolute values. Then the following result immediately follows from Theorem 4.1.

Corollary 4.2 Let $S$ be a nonempty order-metric complete subset of a generalized Archimedean Riesz space. Let $f: S \rightarrow S$ be a self-mapping. Suppose there is a positive number $\alpha<1$, such that

$$
\left|f\left(s_{1}\right)-f\left(s_{2}\right)\right| \preccurlyeq^{X}\left|s_{1}-s_{2}\right|, \quad \text { for all } s_{1}, s_{2} \in S \text {. }
$$

Then $f$ has a unique fixed point.

Next we generalize the concept of order-increasing of mappings on ordered vector spaces.

$\left(S, d_{X}\right)$ and $\left(T, d_{Y}\right)$ be ordered metric spaces, with respect to ordered vector spaces $\left(X, \succcurlyeq^{X}\right)$ and $\left(Y, \succcurlyeq^{Y}\right)$, respectively. A set-valued mapping $F: S \rightarrow 2^{T} \backslash \emptyset$ is said to be ordered metric increasing upward from $S$ to $2^{T} \backslash \emptyset$, whenever there are elements $s^{\prime} \in S, t^{\prime} \in T$ such that, for any given $s_{1}, s_{2} \in S, d_{X}\left(s_{1}, s^{\prime}\right) \preccurlyeq{ }^{X} d_{X}\left(s_{2}, s^{\prime}\right)$ implies that, for every $t_{1} \in F\left(s_{1}\right)$, there is $t_{2} \in F\left(s_{2}\right)$ satisfying $d_{Y}\left(t_{1}, t^{\prime}\right) \preccurlyeq^{Y} d_{Y}\left(t_{2}, t^{\prime}\right)$ (with respect to points $s^{\prime}$ and $\left.t^{\prime}\right) . F$ is said to be ordered metric increasing downward from $S$ to $2^{T} \backslash \emptyset$ (with respect to points $s$ and $t$ ), whenever for any given $s_{1}, s_{2} \in S, d_{X}\left(s_{1}, s^{\prime}\right) \preccurlyeq^{X} d_{X}\left(s_{2}, s^{\prime}\right)$ implies that, for every $t_{2} \in F\left(s_{2}\right)$, there is $t_{1} \in F\left(s_{1}\right)$ satisfying $d_{Y}\left(t_{1}, t^{\prime}\right) \preccurlyeq{ }^{Y} d_{Y}\left(t_{2}, t^{\prime}\right)$. $F$ is said to be ordered metric increasing from $S$ to $2^{T} \backslash \emptyset$ (with respect to points $s^{\prime}$ and $t^{\prime}$ ), whenever $F$ is both ordered metric increasing downward and ordered metric increasing upward. In particular, if $S=T$ and $s^{\prime}=t^{\prime}$, then $F$ is said to be ordered metric increasing (upward, downward) on $S$ (with respect to point $s^{\prime}$ ).

A single-valued mapping $f: S \rightarrow T$ is said to be ordered metric increasing (decreasing) with respect to elements $s^{\prime} \in S, t^{\prime} \in T$, whenever, for any given $s_{1}, s_{2} \in S, d_{X}\left(s_{1}, s^{\prime}\right) \preccurlyeq{ }^{X}$ $d_{X}\left(s_{2}, s^{\prime}\right)$ implies $d_{Y}\left(f\left(s_{1}\right), t^{\prime}\right) \preccurlyeq^{Y} d_{Y}\left(f\left(s_{2}\right), t^{\prime}\right)\left(d_{Y}\left(f\left(s_{1}\right), t^{\prime}\right) \succcurlyeq^{Y} d_{Y}\left(f\left(s_{2}\right), t^{\prime}\right)\right)$.

Definition 4.3 Let $\left(S, d_{X}\right)$ be an ordered metric space with respect to an ordered vector space $\left(X, \succcurlyeq^{X}\right) .\left(S, d_{X}\right)$ is called a monodromy ordered metric space, with respect to an element $s^{\prime} \in S$, whenever the map $d_{X}\left(\cdot, s^{\prime}\right): S \rightarrow X$ is one to one. Such an element $s^{\prime} \in S$ is called a monotonized point of the ordered metric space $\left(S, d_{X}\right)$.

Example 4.4 For an arbitrary positive integer $n$, let $\left(R_{n}, \succcurlyeq_{n}\right)$ denote the ordered vector space, where $n$ is the coordinate ordering on the $n$-dimensional Euclidean space $R_{n}$. Let $S$ be a subset of the positive cone of $\left(R_{n}, \succcurlyeq_{n}\right)$ containing the origin of $R_{n}$. Define an ordered metric $d_{n}$ on $S$ as: for any $x=\left(x_{1}, x_{2}, \ldots, x_{n}\right), y=\left(y_{1}, y_{2}, \ldots, y_{n}\right) \in S$,

$$
d_{n}(x, y)=\left(\left|x_{1}-y_{1}\right|,\left|x_{2}-y_{2}\right|, \ldots,\left|x_{n}-y_{n}\right|\right) .
$$

It follows that

$$
d_{n}(x, 0)=x, \quad \text { for any } x \in S .
$$


It implies that the ordered metric space $\left(S, d_{n}\right)$ is a monodromy ordered metric space with respect to its monotonized point $0 \in S$.

Example 4.5 Let $\left(X, \succcurlyeq^{X}\right)$ be a Riesz space and let the ordered metric $d_{X}$ on $X$ be defined in Example 3.5. Similarly to Example 4.4, we can see that every subset $S$ of the positive cone of $X$ containing the origin of $X$ is a monodromy ordered metric space with the same metric $d_{X}$ on $S$ and with respect to a monotonized point $0 \in S$.

Recall that a nonempty subset $A$ of a poset $(P, \succcurlyeq)$ is said to be inductive if every chain in $A$ has an upper bound in $A$. The next definition extends this concept.

Definition 4.6 A nonempty subset $A$ of a poset $(P, \succcurlyeq)$ is said to be totally inductive in $P$ whenever, for any given chain $\left\{x_{\alpha}\right\} \subset P$, every element $x_{\beta} \in\left\{x_{\alpha}\right\}$ has an upper bound in $A$ implies that the chain $\left\{x_{\alpha}\right\}$ has an upper bound in $A$.

It is clear that every totally inductive subset of a poset is inductive. However, the inverse may not true. The following lemma provides a sufficient condition for an inductive set to be totally inductive.

Lemma 4.7 Let $A$ be an inductive subset of a poset $(P, \succcurlyeq)$. If $A$ has finite number of maximal elements, then $A$ is totally inductive.

Proof Take an arbitrary chain $\left\{x_{\alpha}\right\} \subset P$ satisfying that every element $x_{\beta} \in\left\{x_{\alpha}\right\}$ has an upper bound $z_{\beta} \in A$. We write the set of maximal elements of $A$ by $\left\{u_{1}, u_{2}, \ldots, u_{m}\right\} \subset A$, for some positive integer $m$. We claim that, for any given $x_{\beta} \in\left\{x_{\alpha}\right\}$, we must have

$$
x_{\beta} \preccurlyeq z_{\beta} \preccurlyeq u_{i}, \quad \text { for some } i \text { with } 1 \leq i \leq m \text {. }
$$

To prove (6), assume, by way of contradiction, that (6) does not hold for some $x_{\gamma} \in\left\{x_{\alpha}\right\}$. Then we define

$$
E\left(z_{\gamma}\right)=\left\{z \in A: z \succcurlyeq z_{\gamma}\right\}
$$

Since $z_{\gamma} \in E\left(z_{\gamma}\right)$, we get $E\left(z_{\gamma}\right) \neq \emptyset$. For any arbitrary chain $C$ in $E\left(z_{\gamma}\right) \subset A$, it is also a chain in $A$. It follows that the inductivity of $A$ implies that $C$ has an upper bound $d \in A$. It is clear that $d \in E\left(z_{\gamma}\right)$. Thus, it shows that $d$ is an upper bound of $C$ in $E\left(z_{\gamma}\right)$, hence $E\left(z_{\gamma}\right)$ is inductive. Using Zorn's Lemma, $E\left(z_{\gamma}\right)$ has a maximal element $w$ of $E\left(z_{\gamma}\right)$. In the case if there is $v \in A$ with $v \succcurlyeq w$ and $w \nsucc v$, then $v \in E\left(z_{\gamma}\right)$ and $w$ could not be a maximal element in $E\left(z_{\gamma}\right)$. It shows that $w$ is also a maximal element of $A$. From the hypotheses that $z_{\gamma}$ does not satisfy (6), then the maximal element $w \notin\left\{u_{1}, u_{2}, \ldots, u_{m}\right\}$. It is a contradiction to the assumption that $\left\{u_{1}, u_{2}, \ldots, u_{m}\right\}$ is the collection of all maximal elements of $A$. The claim is proved.

Following (6), a collection of subsets of $C$ is recursively defined as below:

$$
\begin{aligned}
& E_{1}=\left\{x \in\left\{x_{\alpha}\right\}: x \preccurlyeq u_{1}\right\} ; \\
& E_{2}=\left\{x \in\left\{x_{\alpha}\right\} \backslash E_{1}: x \preccurlyeq u_{2}\right\} ;
\end{aligned}
$$




$$
\begin{aligned}
& E_{3}=\left\{x \in\left\{x_{\alpha}\right\} \backslash\left(E_{1} \cup E_{2}\right): x \preccurlyeq u_{3}\right\} ; \\
& \ldots \\
& E_{m-1}=\left\{x \in\left\{x_{\alpha}\right\} \backslash\left(E_{1} \cup E_{2} \cdots E_{m-2}\right): x \preccurlyeq u_{m-1}\right\} ; \\
& E_{m}=\left\{x \in\left\{x_{\alpha}\right\} \backslash\left(E_{1} \cup E_{2} \cdots E_{m-1}\right): x \preccurlyeq u_{m}\right\} .
\end{aligned}
$$

By (6), it follows that $\left\{E_{1}, E_{2}, \ldots, E_{m}\right\}$ is a partition of $\left\{x_{\alpha}\right\}$ with some empty members. Next we show that in $\left\{E_{1}, E_{2}, \ldots, E_{m}\right\}$, there is one and only one nonempty member. To this end, contrarily assume that there are two numbers $1 \leq i<j \leq m$, such that $E_{i}$ and $E_{j}$ both are nonempty. Then we take $x_{\alpha(i)} \in E_{i}$ and $x_{\alpha(j)} \in E_{j}$. Since $\left\{x_{\alpha}\right\}$ is a chain, which is totally ordered, so we must have either $x_{\alpha(i)} \preccurlyeq x_{\alpha(j)}$ or $x_{\alpha(j)} \preccurlyeq x_{\alpha(i)}$. It implies either $x_{\alpha(i)} \in E_{j}$ or $x_{\alpha(j)} \in E_{i}$. It is a contradiction to the fact that $E_{i} \cap E_{j}=\emptyset$. It proves that in $\left\{E_{1}, E_{2}, \ldots, E_{m}\right\}$, there is a unique nonempty member. Thus, this lemma follows immediately.

Theorem 4.8 Let $\left(S, d_{X}\right)$ be a monodromy ordered metric space with respect to an ordered vector space $\left(X, \succcurlyeq^{X}\right)$ and with a monotonized point $s^{\prime} \in S$. Suppose a set-valued mapping $F: S \rightarrow 2^{S} \backslash\{\emptyset\}$ satisfies the following conditions:

1. $F$ is ordered metric increasing upward on $S$ with respect to the point $s^{\prime} \in S$;

2. For every $s \in S$, the set $\left\{d_{X}\left(u, s^{\prime}\right): u \in F(s)\right\}$ is a totally inductive subset of $X$;

3. The set $\bigcup_{s \in S}\left\{d_{X}\left(u, s^{\prime}\right): u \in F(s)\right\}$ is a chain-complete subset of $X$;

4. There is an element $s_{0} \in S$ such that $d_{X}\left(s_{0}, s^{\prime}\right) \preccurlyeq^{X} d_{X}\left(u, s^{\prime}\right)$, for some $u \in F\left(s_{0}\right)$.

Then $F$ has a fixed point.

Proof We define the ordered metric image set for the given mapping $F$ as follows:

$$
A(F)=\left\{d_{X}\left(s, s^{\prime}\right): s \in S \text {, there is } u \in F(s) \text { such that } d_{X}\left(s, s^{\prime}\right) \preccurlyeq^{X} d_{X}\left(u, s^{\prime}\right)\right\} \text {. }
$$

From condition 4, $d_{X}\left(s_{0}, s^{\prime}\right) \in A(F)$; and therefore, $A(F)$ is a nonempty subset of $X$.

For any $d_{X}\left(s, s^{\prime}\right) \in A(F)$, if $u \in F(s)$ satisfying $d_{X}\left(s, s^{\prime}\right) \preccurlyeq^{X} d_{X}\left(u, s^{\prime}\right)$, then from condition 1 and $u \in F(s)$, there is $v \in F(u)$ such that $d_{X}\left(u, s^{\prime}\right) \preccurlyeq^{X} d_{X}\left(v, s^{\prime}\right)$. It implies $d_{X}\left(u, s^{\prime}\right) \in A(F)$. So we get

$$
\begin{aligned}
& d_{X}\left(s, s^{\prime}\right) \in A(F) \quad \text { and } \quad u \in F(s) \text { with } d_{X}\left(s, s^{\prime}\right) \preccurlyeq^{X} d_{X}\left(u, s^{\prime}\right) \\
& \Rightarrow \quad d_{X}\left(u, s^{\prime}\right) \in A(F) .
\end{aligned}
$$

Next, we show that $A(F)$ is inductive.

For any given chain $\left\{x_{\alpha}\right\} \subset A(F)$, suppose that $x_{\alpha}=d_{X}\left(s_{\alpha}, s^{\prime}\right)$ with $u_{\alpha} \in F\left(s_{\alpha}\right)$ such that

$$
d_{X}\left(s_{\alpha}, s^{\prime}\right) \preccurlyeq^{X} d_{X}\left(u_{\alpha}, s^{\prime}\right), \quad \text { for all } \alpha \text {. }
$$

From condition $3, \bigvee\left\{d_{X}\left(s_{\alpha}, s^{\prime}\right)\right\}$ exists and is in $\bigcup_{s \in S}\left\{d_{X}\left(u, s^{\prime}\right): u \in F(s)\right\}$. So there are $\widehat{s} \in S$ and $\widehat{u} \in F(\widehat{s})$ such that

$$
\bigvee\left\{d_{X}\left(s_{\alpha}, s^{\prime}\right)\right\}=d_{X}\left(\widehat{u}, s^{\prime}\right)
$$


It implies

$$
d_{X}\left(s_{\alpha}, s^{\prime}\right) \preccurlyeq^{X} d_{X}\left(\widehat{u}, s^{\prime}\right), \quad \text { for all } \alpha .
$$

For every $\alpha$, since $u_{\alpha} \in F\left(s_{\alpha}\right)$, from condition 1, (10) implies that there is $v_{\alpha} \in F(\widehat{u})$ such that

$$
d_{X}\left(u_{\alpha}, s^{\prime}\right) \preccurlyeq^{X} d_{X}\left(v_{\alpha}, s^{\prime}\right) .
$$

From (8), we have

$$
d_{X}\left(s_{\alpha}, s^{\prime}\right) \preccurlyeq^{X} d_{X}\left(v_{\alpha}, s^{\prime}\right) \quad \text { with } v_{\alpha} \in F(\widehat{u}) \text {, for every } \alpha \text {. }
$$

From condition $2,\left\{d_{X}\left(v, s^{\prime}\right): v \in F(\widehat{u})\right\}$ is a totally inductive subset of $X$. Then (11) implies that the chain $\left\{d_{X}\left(s_{\alpha}, s^{\prime}\right)\right\}$ has an upper bound in $\left\{d_{X}\left(v, s^{\prime}\right): v \in F(\widehat{u})\right\}$, which is denoted by $d_{X}\left(\widehat{v}, s^{\prime}\right)$ with $\widehat{v} \in F(\widehat{u})$. Hence

$$
d_{X}\left(s_{\alpha}, s^{\prime}\right) \preccurlyeq^{X} d_{X}\left(\widehat{v}, s^{\prime}\right), \quad \widehat{v} \in F(\widehat{u}) \text {, for all } \alpha \text {. }
$$

From (9), it implies

$$
d_{X}(\widehat{u}, s)=\bigvee\left\{d_{X}\left(s_{\alpha}, s^{\prime}\right)\right\} \preccurlyeq^{X} d_{X}\left(\widehat{v}, s^{\prime}\right) \quad \text { with } \widehat{v} \in F(\widehat{u})
$$

It follows that $\bigvee\left\{d_{X}\left(s_{\alpha}, s^{\prime}\right)\right\}=d_{X}\left(\widehat{u}, s^{\prime}\right) \in A(F)$. It implies that the chain $\left\{d_{X}\left(s_{\alpha}, s^{\prime}\right)\right\}$ has an upper bound in $A(F)$; and therefore, $A(F)$ is inductive.

Applying Zorn's lemma, $A(F)$ has a maximal element, say $d_{X}\left(x^{*}, s^{\prime}\right)$, for some $s^{*} \in S$, which satisfies

$$
d_{X}\left(s^{*}, s^{\prime}\right) \preccurlyeq^{X} d_{X}\left(u^{*}, s^{\prime}\right), \quad \text { for some } u^{*} \in F\left(s^{*}\right) .
$$

From (7), we have $d_{X}\left(u^{*}, s^{\prime}\right) \in A(F)$. Since $d_{X}\left(s^{*}, s^{\prime}\right)$ is a maximal element of $A(F)$, from (13), it implies that

$$
d_{X}\left(u^{*}, s^{\prime}\right)=d_{X}\left(s^{*}, s^{\prime}\right) .
$$

Since $\left(S, d_{X}\right)$ is monodromy with respect to this element $s^{\prime} \in S$, (14) implies that $s^{*}=u^{*} \in$ $F\left(s^{*}\right)$. Hence $s^{*}$ is a fixed point of $F$.

Recall that every Riesz space (vector lattice) $\left(X, \succcurlyeq^{X}\right)$ can be considered as an ordered metric space with the ordered metric induced by its ordered absolute values. The positive cone of $\left(X, \succcurlyeq^{X}\right)$ is denoted by

$$
X^{+}=\left\{x \in X: x \succcurlyeq^{X} 0\right\} .
$$

Let $S$ be an arbitrary nonempty subset $S$ of $X^{+}$containing 0. From Example 4.5, $\left(S, d_{X}\right)$ is a monodromy ordered metric space with respect to the ordered vector space $\left(X, \succcurlyeq^{X}\right)$, and 
with $0 \in S$ as a monotonized point. It satisfies, for any $s \in S, d_{X}(s, 0)=s$; and therefore, for any $s_{1}, s_{2} \in S, d_{X}\left(s_{1}, 0\right) \preccurlyeq^{X} d_{X}\left(s_{2}, 0\right)$ if and only if $s_{1} \preccurlyeq^{X} s_{2}$. Hence, if a set-valued mapping $F: S \rightarrow 2^{S} \backslash\{\emptyset\}$ is ordered metric increasing upward, with respect to point 0 , then it is ordered increasing upward, that is, whenever for any given $s_{1}, s_{2} \in S, s_{1} \preccurlyeq^{X} s_{2}$ implies that, for every $t_{1} \in F\left(s_{1}\right)$, there is $t_{2} \in F\left(s_{2}\right)$ satisfying $t_{1} \preccurlyeq{ }^{X} t_{2}$. As a consequence of Theorem 4.8, we have the following.

Corollary 4.9 Let $\left(S, d_{X}\right)$ be a nonempty subset of the positive cone of a Riesz space $\left(X, \succcurlyeq^{X}\right)$, which contains 0 . Suppose a set-valued mapping $F: S \rightarrow 2^{S} \backslash\{\emptyset\}$ satisfies the following conditions:

1. $\quad F$ is ordered increasing upward on $S$;

2. for every $s \in S$, the set $F(s)$ is a totally inductive subset of $X$;

3. the set $\bigcup_{s \in S} F(s)$ is a chain-complete subset of $X$;

4. there is an element $s_{0} \in S$ such that $s_{0} \preccurlyeq^{X} u$, for some $u \in F\left(s_{0}\right)$.

Then $F$ has a fixed point.

Remark 4.10 From Lemma 4.7, Theorem 4.8 and Corollary 4.9 still hold if condition 2 is, respectively, replaced by

$2^{\prime}$ For every $s \in S$, the set $\left\{d_{X}\left(u, s^{\prime}\right): u \in F(s)\right\}$, or $F(s)$, is an inductive subset of $X$ with finite number of maximal elements.

Considering single-valued mappings as special cases of set-valued mappings, the following result follows immediately from Theorem 4.8.

Corollary 4.11 Let $\left(S, d_{X}\right)$ be a monodromy ordered metric space with respect to an ordered vector space $\left(X, \succcurlyeq^{X}\right)$ and with a monotonized point $s^{\prime} \in$ S. If a single-valued self-mapping $f$ on $S$ satisfies the following conditions:

1. $f$ is ordered metric increasing on $S$ with respect to the point $s^{\prime} \in S$;

2. The set $\left\{d_{X}\left(f(s), s^{\prime}\right): s \in S\right\}$ is a chain-complete subset of $X$;

3. There is an element $s_{0} \in S$ such that $d_{X}\left(s_{0}, s^{\prime}\right) \preccurlyeq^{X} d_{X}\left(f\left(s_{0}\right), s^{\prime}\right)$.

Then $f$ has a fixed point.

\section{Applications to best ordered approximation problems}

Since Fan [8] proved a best approximation theorem by applying fixed point theorem on normed linear spaces, many authors have extended this theorem to more general topological spaces and have provided applications to approximation theory (see [9-12]). As applications of the fixed point theorems proved in previous section, in this section, we extend the concept of best approximation from metric spaces to ordered metric spaces and give some best ordered approximation theorems.

Definition 5.1 Let $\left(S, d_{X}\right)$ be an ordered metric space with respect to an ordered vector space $\left(X, \succcurlyeq^{X}\right)$ and $K$ a nonempty subset of $S$. Let $f: K \rightarrow S$ be a map. An element $u \in K$ is called a best ordered approximation of $f$ on $K$, if it satisfies

$$
d_{X}(u, f(u))=\min \left\{d_{X}(s, f(u)): s \in K\right\},
$$


where $\min \left\{d_{X}(s, f(u)): s \in K\right\}$ is the smallest (minimum) element of the set $\left\{d_{X}(s, f(u))\right.$ : $s \in K\}$ with respect to the ordering $\succcurlyeq^{X}$ on $X$.

For any given single-valued map $f: K \rightarrow S$, define a set-valued map $T_{f}: K \rightarrow 2^{K}$ by

$$
T_{f}(t)=\left\{v \in K: d_{X}(v, f(t))=\min \left\{d_{X}(s, f(t)): s \in K\right\}\right\}, \quad \text { for all } t \in K \text {. }
$$

This mapping $T_{f}$ is called the approximating mapping of the $\operatorname{map} f$.

It is worthy to note that if $\left(S, d_{X}\right)$ is monodromy, then, for any given map $f: K \rightarrow S$ and for $t \in K$, the value of its approximating mapping $T_{f}(t)$ is either $\emptyset$ or a singleton.

As a consequence of Corollary 4.11, we have the following.

Theorem 5.2 Let $\left(S, d_{X}\right)$ be a monodromy ordered metric space with respect to an ordered vector space $\left(X, \succcurlyeq^{X}\right)$ and with a monotonized point $s^{\prime} \in S$ and $K$ a nonempty order-metric complete subset of $S$ containing $s^{\prime}$. Let $f: K \rightarrow S$ be a map. Suppose the approximating mapping $T_{f}$ off satisfies the following conditions:

1. $T_{f}(s) \neq \emptyset$; and therefore it is a singleton, for every $s \in K$;

2. $T_{f}$ is ordered metric increasing on $K$ with respect to the point $s^{\prime} \in K$;

3. the set $\left\{d_{X}\left(T_{f}\left(s^{\prime}\right)\right): s \in K\right\}$ is a chain-complete subset of $X$;

4. there is an element $s_{0} \in K$ such that $d_{X}\left(s_{0}, s^{\prime}\right) \preccurlyeq{ }^{X} d_{X}\left(f\left(s_{0}\right), s^{\prime}\right)$.

Then $f$ has a best ordered approximation on $K$.

Proof Since $s^{\prime} \in K$, it is easily to see that $\left(K, d_{X}\right)$ is also a monodromy ordered metric space with respect to an ordered vector space $\left(X, \succcurlyeq^{X}\right)$ and with this monotonized points $s^{\prime} \in S$. Condition 1 implies that the map $T_{f}: K \rightarrow S$ is well defined. From conditions $2-4$ in this theorem, the map $T_{f}$ satisfies all conditions in Corollary 4.11. Then it follows that $T_{f}$ has a fixed points $s^{*}=T_{f}\left(s^{*}\right)$. It implies

$$
d_{X}\left(s^{*}, f\left(s^{*}\right)\right)=\min \left\{d_{X}\left(s^{*}, f\left(s^{*}\right)\right): s \in K\right\} .
$$

Hence $s^{*}$ is a best ordered approximation of $f$ on $K$.

Theorem 5.3 Let $\left(S, d_{X}\right)$ be a monodromy ordered metric space, with respect to a generalized Archimedean ordered vector space $\left(X, \succcurlyeq^{X}\right)$ and with a monotonized point $s^{\prime} \in S$ and $K$ a nonempty order-metric complete subset of $S$ containing $s^{\prime}$. Let $f: K \rightarrow S$ be a map. Suppose the approximating mapping $T_{f}: K \rightarrow 2^{K}$ off satisfies the following conditions:

1. $T_{f}(s) \neq \emptyset$, for every $s \in K$;

2. there is a positive number $\alpha<1$, such that $d_{X}\left(T_{f}\left(s_{1}\right), T_{f}\left(s_{2}\right)\right) \preccurlyeq^{X} \alpha d_{X}\left(s_{1}, s_{2}\right)$, for all $s_{1}, s_{2} \in S$.

Then $f$ has a best ordered approximation on $K$.

Proof Since $\left(S, d_{X}\right)$ is monodromy, from condition 1 , it implies that, for every $t \in K$, the value of its approximating mapping $T_{f}(t)$ is a singleton. Hence, we can consider $T_{f}$ as a single-valued map from $K$ to $K$. By condition 2 of this theorem, $T_{f}$ satisfies condition (4) in Theorem 4.1. It follows that $T_{f}$ has a fixed point. Similarly to the proof of Theorem 4.1, the fixed point of $T_{f}$ is a best ordered approximation of $f$ on $K$. 
Definition 5.4 Let $\left(S, d_{X}\right)$ be an ordered metric space with respect to an ordered vector space $\left(X, \succcurlyeq^{X}\right)$ and $K$ a nonempty subset of $S$. Let $f: K \rightarrow S$ be a map. An element $u \in K$ is called an extended best ordered approximation of $f$ on $K$, if it satisfies

$$
d_{X}(u, f(u)) \in \operatorname{Min}\left\{d_{X}(s, f(u)): s \in K\right\}
$$

where $\operatorname{Min}\left\{d_{X}(s, f(u)): s \in K\right\}$ is the set of minimal elements of the set $\left\{d_{X}(s, f(u)): s \in K\right\}$ with respect to the ordering $\succcurlyeq^{X}$ on $X$.

It is clear that even in the case if $\left(S, d_{X}\right)$ is a monodromy, for any given map $f: K \rightarrow S$ and for $t \in K$, the value of its approximating mapping $T_{f}(t)$ may not be a singleton. As a consequence of Theorem 4.8, we have the following.

Theorem 5.5 Let $\left(S, d_{X}\right)$ be a monodromy ordered metric space with respect to an ordered vector space $\left(X, \succcurlyeq^{X}\right)$ and with a monotonized point $s^{\prime} \in S$ and $K$ a nonempty subset of $S$ containing $s^{\prime}$. Let $f: K \rightarrow$ S be a map. Suppose the approximating mapping $T_{f}: K \rightarrow 2^{K}$ of $f$ satisfies the following conditions:

1. $T_{f}(s) \neq \emptyset$, for every $s \in K$;

2. $T_{f}$ is ordered metric increasing upward on $K$ with respect to the point $s^{\prime} \in K$;

3. for every $s \in K$, the set $\left\{d_{X}\left(u, s^{\prime}\right): u \in T_{f}(s)\right\}$ is a totally inductive subset of $X$;

4. the set $\bigcup_{s \in K}\left\{d_{X}\left(u, s^{\prime}\right): u \in T_{f}(s)\right\}$ is a chain-complete subset of $X$;

5. there is an element $s_{0} \in K$ such that $d_{X}\left(s_{0}, s^{\prime}\right) \preccurlyeq^{X} d_{X}\left(u, s^{\prime}\right)$, for some $u \in T_{f}\left(s_{0}\right)$.

Then $f$ has a best ordered approximation on $K$.

\section{Competing interests}

The authors declare that they have no competing interests.

\section{Authors' contributions}

All authors are equal contributors.

\section{Author details}

${ }^{1}$ Department of Mathematics, Shawnee State University, Second Street, Portsmouth, Ohio, USA. ${ }^{2}$ Department of Applied Mathematics, Nanjing University of Economic and Finance, Wenyuan Road, Nanjing, 210046, China. ${ }^{3}$ Department of Applied Mathematics, Nanjing University of Science and Technology, Xiaolingwei Street, Nanjing, Jiangsu 210094, China.

\section{Acknowledgements}

The authors are grateful for those who have offered their valuable suggestions on improving our work.

Received: 8 January 2014 Accepted: 21 April 2014 Published: 06 May 2014

\section{References}

1. Mustafa, Z, Sims, B: A new approach to generalized metric spaces. J. Nonlinear Convex Anal. 7(2), 289-297 (2006)

2. Dhage, BC: Generalized metric space and mapping with fixed point. Bull. Am. Math. Soc. 84, 329-336 (1992)

3. Huang, LG, Zhang, X: Cone metric spaces and fixed point theorems of contractive mappings. J. Math. Anal. Appl. 332, 1468-1476 (2007)

4. Morales, JR, Rojas, S: Cone metric spaces and fixed point theorems of T-contractive mappings. Notas Mat. 4(2), 66-78 (2008)

5. Aliprantis, CD, Burkinshaw, O: Positive Operators. Springer, Berlin (2006)

6. Alber, YI, Reich, S: An iterative method for solving a class of nonlinear operator equations in Banach spaces. Panam. Math. J. 4(2), 39-54 (1994)

7. Carl, S, Heikkilä, S: Fixed Point Theory in Ordered Sets and Applications: From Differential and Integral Equations to Game Theory. Springer, New York (2010)

8. Fan, K: Extensions of two fixed point theorems of F. E. Browder. Math. Z. 112, 234-240 (1969)

9. Lin, TC, Park, S: Approximation and fixed point theorems for condensing composites of multifunctions. J. Math. Anal. Appl. 233, 1-8 (1998)

10. Liu, LS, Li, XK: On approximation theorems and fixed point theorems for non-self-mappings in uniformly convex Banach spaces. Banyan Math. J. 4, 11-20 (1997) 
11. Reich, S: Fixed points in locally convex spaces. Math. Z. 125, 17-31 (1972)

12. Reich, S: Approximate selection sets, best approximations, fixed points, and invariant sets. J. Math. Anal. Appl. 62 , 104-113(1978)

10.1186/1687-1812-2014-109

Cite this article as: Li et al.: Fixed point theorems on ordered vector spaces. Fixed Point Theory and Applications 2014, 2014:109

Submit your manuscript to a SpringerOpen ${ }^{\circ}$ journal and benefit from:

- Convenient online submission

- Rigorous peer review

- Immediate publication on acceptance

Open access: articles freely available online

- High visibility within the field

- Retaining the copyright to your article

Submit your next manuscript at $>$ springeropen.com 